\title{
Influence de la congélation et de la cryodessiccation qui s'ensuit sur le taux de survie et le pourcentage des deux ferments lactiques (culture mixte)
}

\author{
Streptococcus thermophilus et Lactobacillus acidophilus
}

par

A. JABARIT

\section{Introduction}

Dans cette dernière partie de notre étude comparative concernant l'influence des traitements thermiques aux températures basses sur la variation physico-chimique du milieu de culture naturel, le taux de viabilité et le pourcentage respectif des deux ferments lactiques présents sont, à savoir :

- Streptococcus thermophilus.

- Lactobacillus acidophilus.

Les conditions de travail étaient identiques à celles des quatre autres cultures mixtes ; tant pour les températures de congélation que pour les conditions de cryodessiccation.

Les résultats obtenus indiquent la symbiose et l'effet stimulant de la culture mixte, ainsi que la résistance physiologique des ferments lactiques sont différents.

Une étude ultérieure concernera, le comportement de ces cinq cultures mixtes dans des milieux cryoconcentrés d'une part et en présence de substances protectrices et stimulantes d'autre part.

\section{Identification des micro-organismes réactifs utilisés}

Il s'agit des deux ferments lactiques suivants :

- Streptococcus thermophilus,

- Lactobacillus acidophilus,

provenant de l'Ecole Polytechnique Fédérale de Zurich, régénérés et conservés dans notre laboratoire, sous forme de cultures pures et mixtes, à l'état frais et cryodesséchées. 


\section{I. - STREPTOCOCCUS THERMOPHILUS}

\subsection{ORIGINE}

C'est une souche pure de provenance de l'Institut du lait de l'Ecole Polytechnique de Zurich, sous l'immatriculation Streptococcus thermophilus (M.T.I.) 4-4-66.

\subsection{ASPECT MICROSCOPIQUE}

Les ferments lactiques streptocoques thermophiles sont de forme sphérique en chaînettes régulières, d'une dimension de $2 \mu$ environ, présentés toujours deux par deux. Les streptocoques ont une coloration Gram positif.

\subsection{CARActÉRISTIQUES TAXONOMIQUES}

Streptocoque thermophile : c'est un streptocoque homo-fermentatif très répandu dans les produits laitiers, exigeant de la nourriture azotée et vitaminique groupe B et des acides aminés. La fermentation du lactose est un caractère majeur des ferments lactiques.

a) Caractères cultureux

Sur le milieu spécifique Man-Rogosa et Sharpe (M.R.S.) gélifié, les streptocoques donnent de petites colonies ponctifères. Les colonies se forment plus aisément en profondeur qu'en surface.

b) Caractères biochimiques

- Culture à $10^{\circ} \mathrm{C}$ : négatif ; culture à $37^{\circ} \mathrm{C}$ : positif ; culture à $45^{\circ} \mathrm{C}$ : positif ; survie à $63^{\circ} \mathrm{C}$ pendant $30 \mathrm{mn}$ : positif ; fermentation du glucose et production de $\mathrm{CO}_{2}$ : négatif ; réduction rapide du lait tournesolé : négatif (réduction avant coagulation) ; production d'acétone : négatif ; production d'ammoniaque en hydrolysant de l'arginine : négatif ; croissance en présence de chlorure de sodium : 2 p. 100 : négatif, 4 p. 100 : négatif, 6,5 p. 100 : négatif.

- Sucres fermentés : lactose, glucose, galactose, saccharose : positif ; pentose, maltose, momitol, salcine : négatif.

- Non pathogène.

\section{II. - LACTOBACILLUS ACIDOPHILUS}

\subsection{ORIGINE}

C'est un micro-organisme thermophile. Il a été isolé pour la première fois à partir de faeces d'enfants nourris au lait. Bien qu'il ressemble au lactobacille bulgare, il existe des différences taxonomiques très nettes entre ces deux espèces. Le lactobacille acidophile produit de l'acide lactique DL, et fermente une gamme plus étendue de sucres. De plus, il est très résistant à la bile.

La souche est pure de provenance de l'Institut du lait de l'Ecole Fédérale Polytechnique de Zurich, sous l'immatriculation Lactobacil- 
lus acidophilus 3009-9-7-57, réhydratée et régénérée dans notre laboratoire.

\subsection{ASPECT MICROSCOPIQUE}

Les lactobacilles acidophiles sont souvent seules ou par paire sous forme de bâtonnets ou $\mathrm{S}$ (courte chaîne) les extrémités arrondies d'une dimension de 0,6 à $0,9 \times 1,5$ à $6 \mu$ immobiles.

Les lactobacilles ont une coloration Gram positif, les vieilles cultures ont souvent une coloration Gram négatif.

\subsection{Caractéristiques taXonomiques}

Le Lactobacillus acidophilus est biochimiquement bien distinct, à savoir :

- L'acidification dans le lait est faible, de nombreux sucres différents peuvent être assimilés. Cette espèce est la seule à pouvoir s'implanter parmi la flore intestinale des enfants nourris au biberon. On la trouve souvent aussi dans les faeces de l'homme adulte et des mammifères. La possibilité de se multiplier dans l'intestin, pour le lactobacille acidophile est très intéressante.

\section{a) Caractères cultureux}

Sur les milieux spécifiques tels que : Wort Agar ou jus de tomate gélifié, les lactobacilles sur la surface donnent des colonies plus grandes.

Par contre, dans la profondeur ils donnent des petites colonies irrégulières. Ne se développent pas sur le milieu gélatine à $20^{\circ} \mathrm{C}$ et ne produisent pas la liquéfaction de celui-ci. Ne se développent pas sur un milieu naturel comme la pomme de terre.

\section{b) Caractères biochimiques}

- Culture à $15^{\circ} \mathrm{C}$ : négatif ; culture à $20^{\circ} \mathrm{C}-22^{\circ} \mathrm{C}$ : négatif ; culture à $45^{\circ} \mathrm{C}$ : positif ; température optimale : $37^{\circ} \mathrm{C}$; température maximale : $43^{\circ} \mathrm{C}$ à $48^{\circ} \mathrm{C}$; résistance à $60^{\circ} \mathrm{C} / 90 \mathrm{mn}$ : négatif ; résistance à $65^{\circ} \mathrm{C} / 30 \mathrm{mn}$ : négatif ; acide (p. 100) dans le lait : 0,8 ; configuration de l'acide lactique : DL ; production du gaz à partir du sucre : négatif ; production d' $\mathrm{HN}_{3}$, à partir de l'arginine : négatif ; culture en $\mathrm{Na}$ cl 2 p. 100 : positif ; culture en $\mathrm{Na}$ cl 4 p. 100 : positif ; exigences nutritionnelles : riboflavine, acide folique et cyanocobalamine (R.F.S.) ; fermentation des sucres : arabinose et xylose : négatif.

- Fermentation des sucres comme : glucose : positif ; galactose : positif ; lactose : positif ; maltose : positif ; salicine : positif ; saccharose : positif ; tréahalose : positif ; hydrolyse de l'esculine : négatif ; croissance avec 1 p. 100 et 3 p. 100 de bile: positif.

Il s'agit ici du pourcentage de bile liquide et non de bile sèche ou de sels biliaires. La bile employée était une solution à 10 p. 100 
de bile de bovin séchée (oxoid). Le milieu à 1 p. 100 de bile contient donc 0,1 p. 100 de bile sèche.

\section{Travail réalisé}

1) La préparation de milieu de culture se fait à partir du lait écrémé en poudre ayant 14 p. 100 de matière sèche. La quantité est de 1,81 chauffé à l'autoclave $90^{\circ} \mathrm{C}-100^{\circ} \mathrm{C}$ pendant $45 \mathrm{mn}$.

Après l'autoclavage, on refroidit à $45^{\circ} \mathrm{C}$ sous l'eau courante, ce milieu de culture naturelle est prêt pour être ensemencé.

2) La préparation du levain et l'ensemencement, se font avec l'inoculum à partir des souches pures : streptocoque thermophile et lactobacille acidophile âgées de 18 à $24 \mathrm{~h}$. L'inoculum lui-même est conservé à l'état frais au froid à $2^{\circ} \mathrm{C}$ à $4^{\circ} \mathrm{C}$, réchauffé à la température ambiante $\left(22^{\circ} \mathrm{C}-24^{\circ} \mathrm{C}\right)$ juste avant l'ensemencement. La dose d'ensemencement est de 2,5 p. 100 . La durée de l'étuvage est de $1 \mathrm{~h} 30$ à $2 \mathrm{~h}$ à la température de $42^{\circ} \mathrm{C}$.

Laisser refroidir le yoghourt progressivement jusqu'à la phase stationnaire.

Les souches de ferments lactiques elles-mêmes sont conservées dans notre laboratoire à l'état frais et cryodesséchées sous forme de culture pure et mixte.

3) Prélèvement, contrôle physico-chimique et test de viabilité s'effectuent systématiquement, pour mesurer le $\mathrm{pH}$ et l'acidité acide lactique au gramme par litre : connue sous l'appellation en degré Dornic en France, test de viabilité par la méthode de coagulase, ce qui nous permet de déterminer la fin de la phase logarithmique de croissance des ferments lactiques ainsi que l'évolution du $\mathrm{pH}$ et la quantité de l'acide lactique dans le yoghourt.

4) Congélation de culture mixte. Cette phase de l'opération est très importante, car nous cherchons à connaître la résistance de ces souches aux basses températures, à savoir : $-20^{\circ} \mathrm{C},-25^{\circ} \mathrm{C},-30^{\circ} \mathrm{C}$, $-35^{\circ} \mathrm{C}$ et $-40^{\circ} \mathrm{C}$. La vitesse de congélation varie suivant les températures de congélation appliquées entre 3 à $8 \mathrm{~mm}$ à l'heure.

5) Prélèvement, contrôle physico-chimique et surtout le test de viabilité pour déterminer l'action du froid sur le comportement physico-chimique du milieu de culture et en particulier le taux de survie de population et leur pourcentage respectif.

6) CRYOdESSICCATION ET SES DIFFÉRENTES PHASES. Les différentes phases de la cryodessiccation du yoghourt sont les suivantes :

- sublimation $\left\{\begin{array}{l}\text { dessiccation primaire } \\ \text { dessiccation secondaire }\end{array}\right.$ 
Dans cette phase l'eau libre et un certain nombre d'eau liée sont sublimées :

$$
\text { - désorption }\left\{\begin{array}{l}
\text { dessiccation tertiaire } \\
\text { ou } \\
\text { séchage de finition }
\end{array}\right.
$$

Cette phase n'est plus sublimation, c'est une sorte de séchage sous vide. La durée et la température du produit sont très importants. On peut facilement dénaturer la structure hysto-chimique du yoghourt ainsi que provoquer la destruction des cellules vivantes.

La variation du $\mathrm{pH}$ se fait pendant cette période car un certain nombre de substances (lactose, protides) sont touchées. La durée de cette période est de l'ordre de 5-15 mn et la température du produit $30^{\circ} \mathrm{C}-35^{\circ} \mathrm{C}$.

8) DÉTERMINATION DE L'HUMIDITÉ RÉSIDUELLE DES PRODUITS CRYODESSÉcHÉs. La détermination de l'humidité résiduelle dans les produits cryodesséchés est très importante, car le pourcentage d'eau résiduelle prouve la qualité du produit cryodesséché. En particulier le taux de survie de la population (ferments lactiques) ainsi que la dégradation hysto-chimique au cours du veillissement du produit. La durée de conservation, les qualités des produits cryodesséchés dépendent du pourcentage de l'humidité résiduelle. Dans le cas où celui-ci est supérieur à 4 p. 100 la durée de conservation du produit cryodesséché ne peut pas dépasser plus de six mois. L'humidité entre 1 à 2 p. 100 permet la conservation de denrée alimentaire (yoghourt) plus d'un an. La déshydratation trop poussée au-dessous de 1 p. 100 n'est pas souhaitable pour un yoghourt cryodesséché. Le yoghourt cryodesséché est mis en emballage sous vide et compensé à l'azote sec.

9) Test de RÉHydratation, analyses organoleptiques. D'après les tests de réhydratation et les analyses organoleptiques qui se font d'une façon systématique, le yoghourt cryodesséché réhydraté : (14 g du produit ramenés à 100 dans l'eau tiède à $45^{\circ} \mathrm{C}$ et étuvé à $42^{\circ} \mathrm{C}$ pendant 2 à $3 \mathrm{~h}$ ), refroidi à $4^{\circ} \mathrm{C}$ dans le réfrigérateur, il est prêt à déguster le lendemain. Le produit à déguster est réchauffé à la température de l'ambiance $\left(20^{\circ} \mathrm{C}\right)$. L'aspect général du produit réhydraté est satisfaisant, à savoir : la couleur est blanche, la texture est granuleuse ou lisse plus ou moins ferme, souvent, il ressemble au yoghourt dit bulgare. Au cas où ce yoghourt serait présenté sous forme aromatisé, il serait impossible de distinguer entre un yoghourt cryodesséché-réhydraté et celui du frais aromatisé.

Par conséquent, dans l'immédiat, il est intéressant d'envisager dans un premier temps de faire du yoghourt cryodesséché aux différents parfums ou tout simplement plus ou moins sucré et fruité. 
Durée de la cryodessiccation suivant la température de chauffage

(température de congélation à $-20^{\circ} \mathrm{C}$ )

\begin{tabular}{|c|c|c|c|c|c|}
\hline \multirow{2}{*}{$\begin{array}{l}\text { Température de } \\
\text { chauffage }\end{array}$} & \multicolumn{2}{|c|}{ Dessiccation } & \multirow{2}{*}{ Désorption } & \multirow{2}{*}{$\begin{array}{l}\text { Durée du } \\
\text { cycle } \\
\text { en heures }\end{array}$} & \multirow{2}{*}{ Observations } \\
\hline & primaire & secondaire & & & \\
\hline $80^{\circ} \mathrm{C}$ & $7 \mathrm{~h}$ & $3 \mathrm{~h}$ & $10 \mathrm{mn}$ & $10 \mathrm{~h} 10$ & \\
\hline $90^{\circ} \mathrm{C}$ & $7 \mathrm{~h}$ & $2 \mathrm{~h}$ & $30 \mathrm{mn}$ & 9 h 30 & \\
\hline $100^{\circ} \mathrm{C}$ & $7 \mathrm{~h}$ & $40 \mathrm{mn}$ & $7 \mathrm{mn}$ & 7 h 47 & \\
\hline $110^{\circ} \mathrm{C}$ & 5 h 20 & 3 h 10 & $15 \mathrm{mn}$ & 8 h 45 & \\
\hline $120^{\circ} \mathrm{C}$ & 4 h 20 & $1 \mathrm{~h} 15$ & $40 \mathrm{mn}$ & $6 \mathrm{~h} 15$ & \\
\hline \multicolumn{6}{|c|}{ 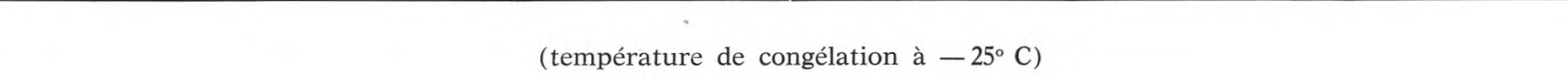 } \\
\hline \multirow{2}{*}{$\begin{array}{l}\text { Température de } \\
\text { chauffage }\end{array}$} & \multicolumn{2}{|c|}{ Dessiccation } & \multirow{2}{*}{ Désorption } & \multirow{2}{*}{$\begin{array}{l}\text { Durée du } \\
\text { cycle } \\
\text { en heures }\end{array}$} & \multirow{2}{*}{ Observations } \\
\hline & primaire & secondaire & & & \\
\hline $80^{\circ} \mathrm{C}$ & $6 \mathrm{~h} 50$ & 2 h 40 & 10 & 8 h 10 & \\
\hline $90^{\circ} \mathrm{C}$ & $6 \mathrm{~h}$ & 2 h 30 & 5 & 8 h 35 & \\
\hline $100^{\circ} \mathrm{C}$ & 5 h 20 & $1 \mathrm{~h} 40$ & 15 & 7 h 05 & \\
\hline $110^{\circ} \mathrm{C}$ & 6 h 15 & 50 & 5 & 7 h 10 & \\
\hline $120^{\circ} \mathrm{C}$ & $4 \mathrm{~h}$ & $2 \mathrm{~h} 50$ & 20 & $7 \mathrm{~h} 10$ & \\
\hline
\end{tabular}


Durée de la cryodessiccation suivant la température de chauffage

(température de congélation à $-30^{\circ} \mathrm{C}$ )

\begin{tabular}{|c|c|c|c|c|c|}
\hline \multirow{2}{*}{$\begin{array}{l}\text { Température de } \\
\text { chauffage }\end{array}$} & \multicolumn{2}{|c|}{ Dessiccation } & \multirow{2}{*}{ Désorption } & \multirow{2}{*}{$\begin{array}{c}\text { Durée du } \\
\text { cycle } \\
\text { en heures }\end{array}$} & \multirow{2}{*}{ Observations } \\
\hline & primaire & secondaire & & & \\
\hline $80^{\circ} \mathrm{C}$ & $5 \mathrm{~h} 20$ & $1 \mathrm{~h} 40$ & $30 \mathrm{mn}$ & $8 \mathrm{~h} 30$ & \\
\hline $90^{\circ} \mathrm{C}$ & $6 \mathrm{~h} 40$ & $2 \mathrm{~h}$ & $20 \mathrm{mn}$ & $9 \mathrm{~h}$ & \\
\hline $100^{\circ} \mathrm{C}$ & $6 \mathrm{~h} 20$ & $2 \mathrm{~h} 10$ & $10 \mathrm{mn}$ & 8 h 40 & \\
\hline $110^{\circ} \mathrm{C}$ & $5 \mathrm{~h}$ & $2 \mathrm{~h}$ & $10 \mathrm{mn}$ & $7 \mathrm{~h} 10$ & \\
\hline $120^{\circ} \mathrm{C}$ & $5 \mathrm{~h} 10$ & $2 \mathrm{~h}$ & $5 \mathrm{mn}$ & 7 h 15 & \\
\hline \multicolumn{6}{|c|}{ (température de congélation à $-35^{\circ} \mathrm{C}$ ) } \\
\hline \multirow{2}{*}{$\begin{array}{l}\text { Température de } \\
\text { chauffage }\end{array}$} & \multicolumn{2}{|c|}{ Dessiccation } & \multirow{2}{*}{ Désorption } & \multirow{2}{*}{$\begin{array}{l}\text { Durée du } \\
\text { cycle } \\
\text { en heures }\end{array}$} & \multirow{2}{*}{ Observations } \\
\hline & primaire & secondaire & & & \\
\hline $80^{\circ} \mathrm{C}$ & $8 \mathrm{~h}$ & $2 \mathrm{~h}$ & $15 \mathrm{mn}$ & $10 \mathrm{~h} 15$ & \\
\hline $90^{\circ} \mathrm{C}$ & $8 \mathrm{~h}$ & $4 \mathrm{mn}$ & $10 \mathrm{mn}$ & 8 h 50 & \\
\hline $100^{\circ} \mathrm{C}$ & $6 \mathrm{~h} 30$ & $1 \mathrm{~h} 40$ & $20 \mathrm{mn}$ & 8 h 30 & \\
\hline $110^{\circ} \mathrm{C}$ & $6 \mathrm{~h} 10$ & $1 \mathrm{~h} 15$ & $5 \mathrm{mn}$ & 7 h 30 & \\
\hline $120^{\circ} \mathrm{C}$ & $4 \mathrm{~h} 20$ & $3 \mathrm{~h}$ & $20 \mathrm{mn}$ & 7 h 20 & \\
\hline
\end{tabular}


Durée de la cryodessiccation suivant la température du chauffage

(température de congélation à $-40^{\circ} \mathrm{C}$ )

\begin{tabular}{|c|c|c|c|c|c|}
\hline \multirow{2}{*}{$\begin{array}{l}\text { Température de } \\
\text { chauffage }\end{array}$} & \multicolumn{2}{|c|}{ Dessiccation } & \multirow{2}{*}{ Désorption } & \multirow{2}{*}{$\begin{array}{l}\text { Durée du } \\
\text { cycle } \\
\text { en heures }\end{array}$} & \multirow{2}{*}{ Observations } \\
\hline & primaire & secondaire & & & \\
\hline $80^{\circ} \mathrm{C}$ & $6 \mathrm{~h} 30$ & $1 \mathrm{~h} 45$ & $20 \mathrm{mn}$ & 8 h 35 & \\
\hline $90^{\circ} \mathrm{C}$ & $8 \mathrm{~h}$ & $1 \mathrm{~h}$ & $10 \mathrm{mn}$ & $9 \mathrm{~h} 10$ & \\
\hline $100^{\circ} \mathrm{C}$ & $6 \mathrm{~h} 30$ & $2 \mathrm{~h} 30$ & $15 \mathrm{mn}$ & 9 h 15 & \\
\hline $110^{\circ} \mathrm{C}$ & 5 h 30 & $2 \mathrm{~h} 50$ & $20 \mathrm{mn}$ & $8 \mathrm{~h} 10$ & \\
\hline $120^{\circ} \mathrm{C}$ & 5 h 30 & $1 \mathrm{~h} 30$ & $15 \mathrm{mn}$ & 7 h 15 & \\
\hline
\end{tabular}


Voici quelques exemples de la variation du $\mathrm{pH}$ du yoghourt au cours de la cryodessiccation.

+ la température de congélation pour ces yaourts qui était de $-20^{\circ} \mathrm{C}$

\begin{tabular}{|c|c|c|c|c|c|c|}
\hline Température de chauffage & $80^{\circ} \mathrm{C}$ & $90^{\circ} \mathrm{C}$ & $100^{\circ} \mathrm{C}$ & $110^{\circ} \mathrm{C}$ & $120^{\circ} \mathrm{C}$ & Observations \\
\hline Le $\mathrm{pH}$ du yaourt frais & 4,3 & 4,6 & 4,3 & 4,5 & 4,4 & \\
\hline $\begin{array}{c}\text { Le pH du yaourt cryodesséché } \\
\text { réhydraté }\end{array}$ & 4,2 & 4,5 & 4,2 & 4,4 & 4,3 & \\
\hline
\end{tabular}

+ la température de congélation pour ces yaourts qui était de $-25^{\circ} \mathrm{C}$

\begin{tabular}{|c|c|c|c|c|c|c|}
\hline Température de chauffage & $80^{\circ} \mathrm{C}$ & $90^{\circ} \mathrm{C}$ & $100^{\circ} \mathrm{C}$ & $110^{\circ} \mathrm{C}$ & $120^{\circ} \mathrm{C}$ & Observations \\
\hline Le $\mathrm{pH}$ du yaourt frais & 4,60 & 4,70 & 4,40 & 4,25 & 4,8 & \\
\hline $\begin{array}{c}\text { Le pH du yaourt cryodesséché } \\
\text { réhydraté }\end{array}$ & 4,55 & 4,60 & 4,35 & 4,20 & 4,7 & \\
\hline
\end{tabular}


+ la température de congélation pour ces yaourts qui était de $-30^{\circ} \mathrm{C}$

\begin{tabular}{c|c|c|c|c|c}
\hline Température de chauffage & $80^{\circ} \mathrm{C}$ & $90^{\circ} \mathrm{C}$ & $100^{\circ} \mathrm{C}$ & $110^{\circ} \mathrm{C}$ & $120^{\circ} \mathrm{C}$ \\
\hline $\begin{array}{c}\text { Le pH du yaourt frais } \\
\text { Le pH du yaourt cryodesséché } \\
\text { réhydraté }\end{array}$ & 4,50 & 4,25 & 4,25 & 4,40 & 4,45 \\
\hline
\end{tabular}

+ la température de congélation pour ces yaourts qui était de $-35^{\circ} \mathrm{C}$

\begin{tabular}{c|c|c|c|c|c}
\hline Température de chauffage & $80^{\circ} \mathrm{C}$ & $90^{\circ} \mathrm{C}$ & $100^{\circ} \mathrm{C}$ & $110^{\circ} \mathrm{C}$ & $120^{\circ} \mathrm{C}$ \\
\hline $\begin{array}{c}\text { Le pH du yaourt frais } \\
\text { Le pH du yaourt cryodesséché } \\
\text { réhydraté }\end{array}$ & 4,35 & 4,40 & 4,30 & 4,25 & 4,50 \\
\hline
\end{tabular}

+ la température de congélation de ces yaourts qui était de $-40^{\circ} \mathrm{C}$

\begin{tabular}{c|c|c|c|c|c}
\hline Température de chauffage & $80^{\circ} \mathrm{C}$ & $90^{\circ} \mathrm{C}$ & $100^{\circ} \mathrm{C}$ & $110^{\circ} \mathrm{C}$ & $120^{\circ} \mathrm{C}$ \\
\hline $\begin{array}{c}\text { Le pH du yaourt frais } \\
\text { Le pH du yaourt cryodesséché } \\
\text { réhydraté }\end{array}$ & 4,30 & 4,25 & 4,30 & 4,25 & 4,20 \\
\hline & 4,20 & 4,20 & 4,20 & 4,20 & 4,10 \\
\hline
\end{tabular}




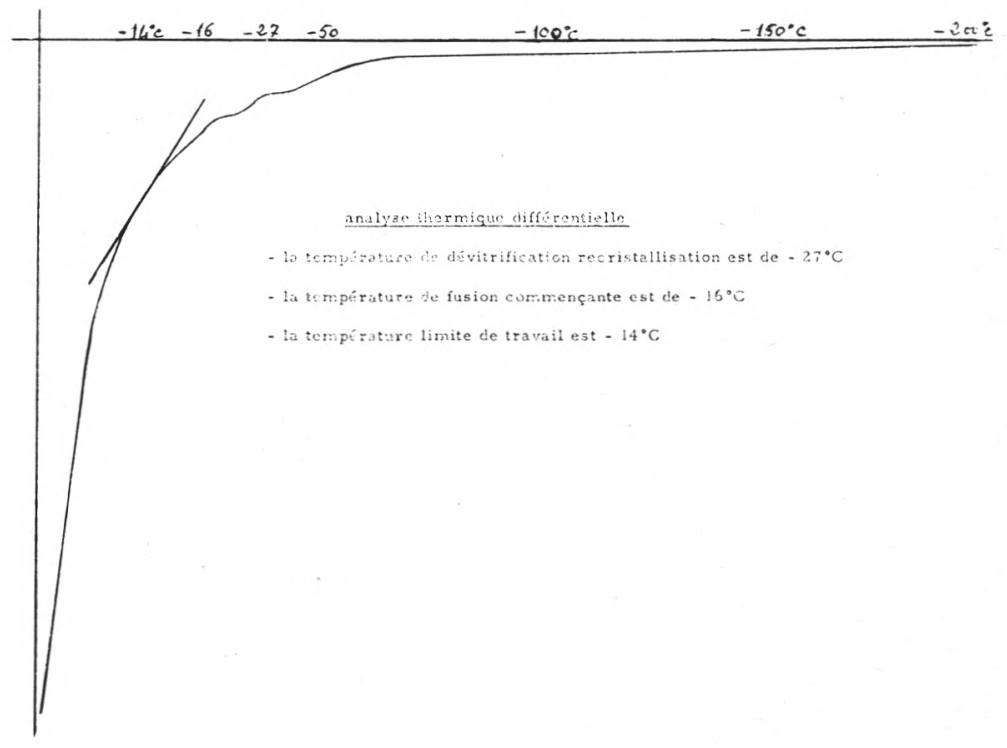

fig. 1

Yoghourt à base de ferments lactiques:

- Streptocoque thermophile.

- Lactobacille acidophile. 
Influence de la température de congélation $-20^{\circ} \mathrm{C}$ et la cryodessiccation qui s'ensuit sur le taux de survie des deux ferments lactiques :

Streptococcus thermophilus et Lactobacillus acidophilus

\begin{tabular}{|c|c|c|c|c|c|c|c|}
\hline \multicolumn{2}{|c|}{ Température de chauffage en ${ }^{\circ} \mathrm{C}$} & 80 & 90 & 100 & 110 & 120 & Observations \\
\hline $\begin{array}{l}\text { Nombre des ferments } \\
\text { lactiques (culture } \\
\text { mixte) viables par ml }\end{array}$ & $\begin{array}{c}\text { frais } \\
\text { congelé } \\
\text { à } \\
-20^{\circ} \mathrm{C} \\
\text { cryo- } \\
\text { desséché }\end{array}$ & $10^{9}$ & $\begin{array}{l}10^{9} \\
10^{9}\end{array}$ & $10^{10}$ & $10^{9}$ & $10^{10}$ & $\begin{array}{l}\text { La méthode de contrôle de } \\
\text { viabilité des ferments lacti- } \\
\text { ques est basée sur le Nom- } \\
\text { bre le Plus Probable (N.P.P.) } \\
\text { Hoskins. }\end{array}$ \\
\hline
\end{tabular}

Le nombre des ferments lactiques viables dans le yaourt frais est de $10^{10}$.

Le nombre des ferments lactiques viables dans le yaourt congelé à $-40^{\circ} \mathrm{C}$ est de...

Le nombre des ferments lactiques viables dans le yaourt cryodesséché est de $10^{\circ}$. 
Influence de la température de congélation $-20^{\circ} \mathrm{C}$ ct de la cryodessiccation qui s'ensuit sur le pourcentage des deux ferments lactiques (culture mixte) :

Streptococcus thermophilus et Lactobacillus acidophilus

\begin{tabular}{|c|c|c|c|c|c|c|c|c|}
\hline \multicolumn{3}{|c|}{ Température de chauffage en ${ }^{\circ} \mathrm{C}$} & 80 & 90 & 100 & 110 & 120 & Observations \\
\hline 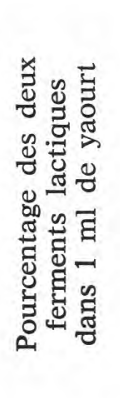 & $\begin{array}{c}\text { frais } \\
\text { congelé } \\
\text { à } \\
-20^{\circ} \mathrm{C} \\
\text { cryo- } \\
\text { desséché }\end{array}$ & $\begin{array}{l}\text { strepto. } \\
\text { lacto. } \\
\text { strepto. } \\
\text { lacto. } \\
\text { strepto. } \\
\text { lacto. }\end{array}$ & $\begin{array}{l}55 \\
45\end{array}$ & $\begin{array}{l}50 \\
50 \\
56 \\
44\end{array}$ & $\begin{array}{l}50 \\
50\end{array}$ & $\begin{array}{l}57 \\
43\end{array}$ & $\begin{array}{l}50 \\
50 \\
58 \\
42 \\
53 \\
47\end{array}$ & $\begin{array}{l}\text { - La méthode employée est l'exa- } \\
\text { men microscopique sur les frot- } \\
\text { tis colorés au bleu de méthy- } \\
\text { lène. } \\
\text { - Un contrôle parallèle par la mé- } \\
\text { thode de numération globale } \\
\text { avec le mélangeur de Potain et } \\
\text { la cellule de Malassez et Vignal } \\
\text { a été effectué. }\end{array}$ \\
\hline
\end{tabular}

- Le pourcentage moyen des deux ferments lactiques respectifs est de 50 p. 100 pour le streptocoque et 50 p. 100 pour le lactobacille, dans la culture mixte fraîche.

- Le pourcentage moyen de ces deux ferments lactiques respectifs (culture mixte) congelés à - $20^{\circ} \mathrm{C}$ est de $56 \mathrm{p}$. 100 pour le streptocoque et 44 p. 100 pour le lactobacille.

- Le pourcentage moyen de ces deux ferments lactiques respectifs cryodesséchés réhydratés est de 52 p. 100 pour le streptocoque et 48 p. 100 pour le lactobacille. 
Influence de la température de congélation $-25^{\circ} \mathrm{C}$ et la cryodessiccation qui s'ensuit sur le taux de survie des deux ferments lactiques :

Streptococcus thermophilus et Lactobacillus acidophilus

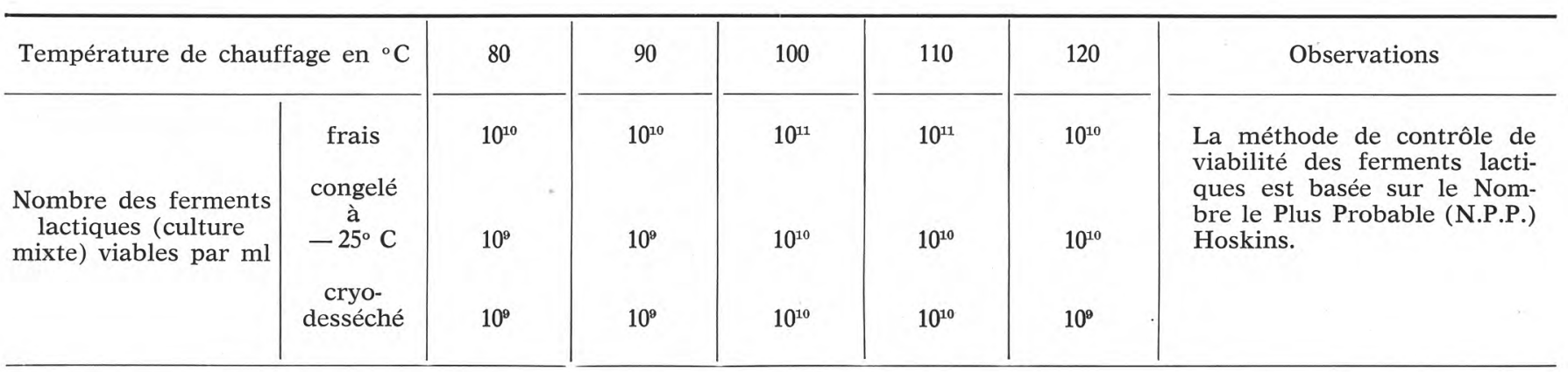

Le nombre des ferments lactiques viables dans le yaourt frais est de $10^{10}$.

Le nombre des ferments lactiques viables dans le yaourt congelé à $-40^{\circ} \mathrm{C}$ et de $-25^{\circ} \mathrm{C}$ est de $10^{\circ}$.

Le nombre des ferments lactiques viables dans le yaourt cryodesséché est de $10^{9}$. 
Influence de la température de congélation $-25^{\circ} \mathrm{C}$ et de la cryodessiccation qui s'ensuit sur le pourcentage des deux ferments lactiques (culture mixte) :

Streptococcus thermophilus et Lactobacillus acidophilus

\begin{tabular}{|c|c|c|c|c|c|c|c|c|}
\hline \multicolumn{3}{|c|}{ Température de chauffage en ${ }^{\circ} \mathrm{C}$} & 80 & 90 & 100 & 110 & 120 & Observations \\
\hline 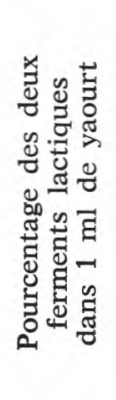 & $\begin{array}{c}\text { frais } \\
\text { congelé } \\
\text { à } \\
-25^{\circ} \mathrm{C} \\
\text { cryo- } \\
\text { desséché }\end{array}$ & $\begin{array}{c}\text { strepto. } \\
\text { lacto. } \\
\text { strepto. } \\
\text { lacto. } \\
\text { strepto. } \\
\text { lacto. }\end{array}$ & $\begin{array}{l}52 \\
48 \\
54 \\
46\end{array}$ & $\begin{array}{l}54 \\
46 \\
55 \\
45\end{array}$ & $\begin{array}{l}52 \\
48 \\
59 \\
41\end{array}$ & $\begin{array}{l}51 \\
49 \\
57 \\
43\end{array}$ & $\begin{array}{l}53 \\
47 \\
53 \\
47 \\
45 \\
55 \\
45\end{array}$ & $\begin{array}{l}\text { - La méthode employée est l'exa- } \\
\text { men microscopique sur les frot- } \\
\text { tis colorés au bleu de méthy- } \\
\text { lène. } \\
\text { - Un contrôle parallèle par la mé- } \\
\text { thode de numération globale } \\
\text { avec le mélangeur de Potain et } \\
\text { la cellule de Malassez et Vignal } \\
\text { a été effectué. }\end{array}$ \\
\hline
\end{tabular}

- Le pourcentage moyen des deux ferments lactiques respectifs est de 52,4 p. 100 pour le streptocoque et de 47,6 p. 100 pour le lactobacille, dans la culture mixte fraîche.

- Le pourcentage moyen de ces deux ferments lactiques respectifs (culture mixte) congelés à $-25^{\circ} \mathrm{C}$ est de $55,6 \mathrm{p}$. 100 pour le streptocoque et 44,4 p. 100 pour le lactobacille.

- Le pourcentage moyen de ces deux ferments lactiques respectifs cryodesséchés réhydratés est de 53,8 p. 100 pour le streptocoque et 46,2 p. 100 pour le lactobacille. 
Influence de la température de congélation $-30^{\circ} \mathrm{C}$ et la cryodessiccation qui s'ensuit sur le taux de survie des deux ferments lactiques :

Streptococcus thermophilus et Lactobacillus acidophilus

\begin{tabular}{|c|c|c|c|c|c|c|c|}
\hline \multicolumn{2}{|c|}{ Température de chauffage en ${ }^{\circ} \mathrm{C}$} & 80 & 90 & 100 & 110 & 120 & Observations \\
\hline $\begin{array}{l}\text { Nombre des ferments } \\
\text { lactiques (culture } \\
\text { mixte) viables par ml }\end{array}$ & $\begin{array}{c}\text { frais } \\
\text { congelé } \\
\text { à } \\
-30^{\circ} \mathrm{C} \\
\text { cryo- } \\
\text { desséché }\end{array}$ & $\begin{array}{l}10^{10} \\
10^{10}\end{array}$ & $10^{10}$ & $\begin{array}{l}10^{10} \\
10^{10}\end{array}$ & $\begin{array}{l}10^{10} \\
10^{10}\end{array}$ & $\begin{array}{l}10^{10} \\
10^{10}\end{array}$ & $\begin{array}{l}\text { La méthode de contrôle de } \\
\text { viabilité des ferments lacti- } \\
\text { ques est basée sur le Nom- } \\
\text { bre le Plus Probable (N.P.P.) } \\
\text { Hoskins. }\end{array}$ \\
\hline
\end{tabular}

Le nombre des ferments lactiques viables dans le yaourt frais est de $10^{11}$ par ml.

Le nombre des ferments lactiques viables dans le yaourt congelé à $-30^{\circ} \mathrm{C}$ est de $10^{10}$ par $\mathrm{ml}$.

Le nombre des ferments lactiques viables dans le yaourt cryodesséché est de $10^{10}$ par $\mathrm{ml}$. 
Influence de la température de congélation $-30^{\circ} \mathrm{C}$ et de la cryodessiccation qui s'ensuit sur le pourcentage

des deux ferments lactiques (culture mixte) :

Streptococcus thermophilus et Lactobacillus acidophilus

\begin{tabular}{|c|c|c|c|c|c|c|c|c|}
\hline \multicolumn{3}{|c|}{ Température de chauffage en ${ }^{\circ} \mathrm{C}$} & 80 & 90 & 100 & 110 & 120 & Observations \\
\hline 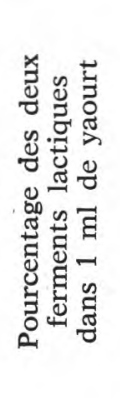 & $\begin{array}{c}\text { frais } \\
\text { congelé } \\
\text { à } \\
-30^{\circ} \mathrm{C} \\
\text { cryo- } \\
\text { desséché }\end{array}$ & $\begin{array}{l}\text { strepto. } \\
\text { lacto. } \\
\text { strepto. } \\
\text { lacto. } \\
\text { strepto. } \\
\text { lacto. }\end{array}$ & $\begin{array}{l}51 \\
49 \\
57 \\
43 \\
54 \\
46\end{array}$ & $\begin{array}{l}51 \\
49 \\
57 \\
43\end{array}$ & $\begin{array}{l}50 \\
50 \\
59 \\
41 \\
52 \\
48\end{array}$ & $\begin{array}{l}50 \\
50 \\
56 \\
44 \\
52 \\
48\end{array}$ & $\begin{array}{l}50 \\
50 \\
56 \\
44 \\
53 \\
47\end{array}$ & $\begin{array}{l}\text { - La méthode employée est l'exa- } \\
\text { men microscopique sur les frot- } \\
\text { tis colorés au bleu de méthy- } \\
\text { lène. } \\
\text { - Un contrôle parallèle par la mé- } \\
\text { thode de numération globale } \\
\text { avec le mélangeur de Potain et } \\
\text { la cellule de Malassez et Vignal } \\
\text { a été effectué. }\end{array}$ \\
\hline
\end{tabular}

- Le pourcentage moyen des deux ferments lactiques respectifs est de 50 p. 100 pour le streptocoque et 50 p. 100 pour le lactobacille, dans la culture mixte fraîche.

- Le pourcentage moyen de ces deux ferments lactiques respectifs (culture mixte) congelés à - $30^{\circ} \mathrm{C}$ est de $57 \mathrm{p}$. 100 pour le streptocoque et 43 p. 100 pour le lactobacille.

- Le pourcentage moyen de ces deux ferments lactiques respectifs cryodesséchés réhydratés est de 52,8 p. 100 pour le streptocoque et 47,2 p. 100 pour le lactobacille. 
Influence de la température de congélation $-35^{\circ} \mathrm{C}$ et la cryodessiccation qui s'ensuit sur le taux de survie des deux ferments lactiques:

Streptococcus thermophilus et Lactobacillus acidophilus

\begin{tabular}{|c|c|c|c|c|c|c|c|}
\hline \multicolumn{2}{|c|}{ Température de chauffage en ${ }^{\circ} \mathrm{C}$} & 80 & 90 & 100 & 110 & 120 & Observations \\
\hline $\begin{array}{l}\text { Nombre des ferments } \\
\text { lactiques (culture } \\
\text { mixte) viables par ml }\end{array}$ & $\begin{array}{c}\text { frais } \\
\text { congelé } \\
\text { à } \\
-35^{\circ} \mathrm{C} \\
\text { cryo- } \\
\text { desséché }\end{array}$ & $\begin{array}{l}10^{9} \\
10^{9}\end{array}$ & $\begin{array}{l}10^{9} \\
10^{8}\end{array}$ & $\begin{array}{l}10^{9} \\
10^{9}\end{array}$ & $\begin{array}{l}10^{10} \\
10^{10}\end{array}$ & $\begin{array}{l}10^{10} \\
10^{8}\end{array}$ & $\begin{array}{l}\text { La méthode de contrôle de } \\
\text { viabilité des ferments lacti- } \\
\text { ques est basée sur le Nom- } \\
\text { bre le Plus Probable (N.P.P.) } \\
\text { Hoskins. }\end{array}$ \\
\hline
\end{tabular}

Le nombre des ferments lactiques viables dans le yaourt frais est de...

Le nombre des ferments lactiques viables dans le yaourt congelé à $-35^{\circ} \mathrm{C}$ est de...

Le nombre des ferments lactiques viables dans le yaourt cryodesséché est de... 
Influence de la température de congélation $-35^{\circ} \mathrm{C}$ et de la cryodessiccation qui s'ensuit sur le pourcentage

des deux ferments lactiques (culture mixte) :

Streptococcus thermophilus et Lactobacillus acidophilus

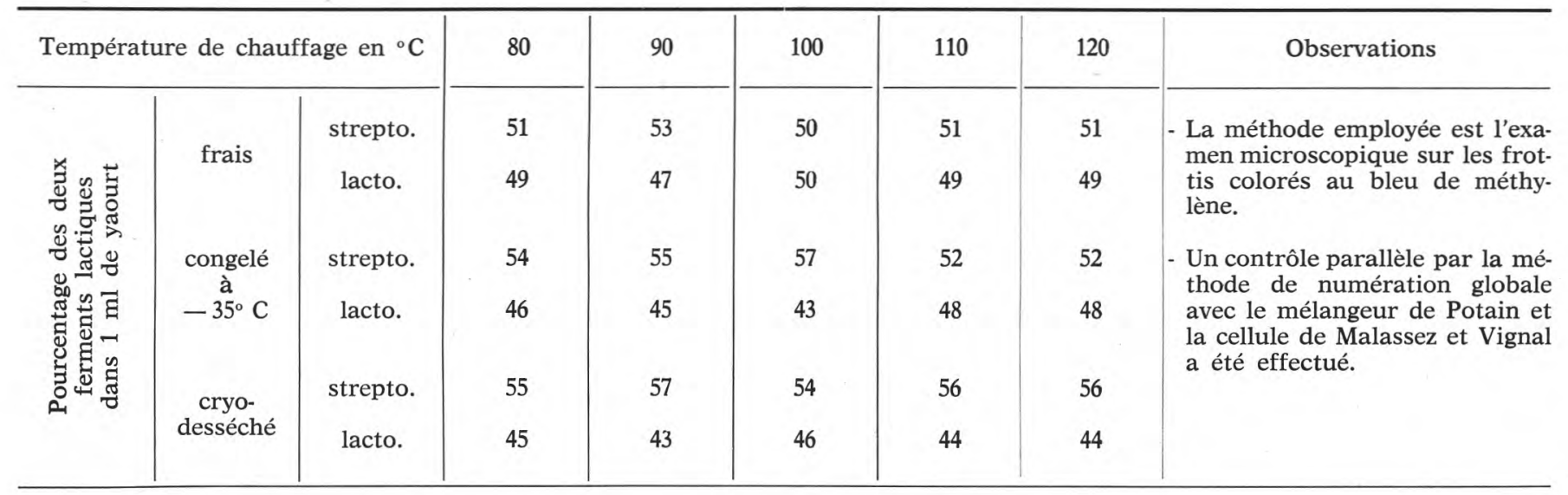

- Le pourcentage moyen des deux ferments lactiques respectifs est de... pour le streptocoque et pour le lactobacille, dans la culture mixte fraîche.

- Le pourcentage moyen de ces deux ferments lactiques respectifs (culture mixte) congelés à... est de... pour le streptocoque et... pour le lactobacille.

- Le pourcentage moyen de ces deux ferments lactiques respectifs cryodesséchés réhydratés est de... pour le streptocoque et... pour le lactobacille. 
Influence de la température de congélation $-40^{\circ} \mathrm{C}$ et la cryodessiccation qui s'ensuit sur le taux de survie des deux ferments lactiques :

Streptococcus thermophilus et Lactobacillus acidophilus

\begin{tabular}{|c|c|c|c|c|c|c|c|}
\hline \multicolumn{2}{|c|}{ Température de chauffage en ${ }^{\circ} \mathrm{C}$} & 80 & 90 & 100 & 110 & 120 & Observations \\
\hline $\begin{array}{l}\text { Nombre des ferments } \\
\text { lactiques (culture } \\
\text { mixte) viables par ml }\end{array}$ & $\begin{array}{c}\text { frais } \\
\text { congelé } \\
\text { à } \\
-40^{\circ} \mathrm{C} \\
\text { cryo- } \\
\text { desséché }\end{array}$ & $10^{9}$ & $10^{10}$ & $10^{10}$ & $10^{10}$ & $\begin{array}{l}10^{10} \\
10^{9}\end{array}$ & $\begin{array}{l}\text { La méthode de contrôle de } \\
\text { viabilité des ferments lacti- } \\
\text { ques est basée sur le Nom- } \\
\text { bre le Plus Probable (N.P.P.) } \\
\text { Hoskins. }\end{array}$ \\
\hline
\end{tabular}

Le nombre des ferments lactiques viables dans le yaourt frais est de $10^{10}$ par ml.

Le nombre des ferments lactiques viables dans le yaourt congelé à $-40^{\circ} \mathrm{C}$ est de $10^{9} \mathrm{par} \mathrm{ml}$.

Le nombre des ferments lactiques viables dans le yaourt cryodesséché est de $10^{9}$ par ml. 
des deux ferments lactiques (culture mixte) :

Streptococcus thermophilus et Lactobacillus acidophilus

\begin{tabular}{|c|c|c|c|c|c|c|c|c|}
\hline \multicolumn{3}{|c|}{ Température de chauffage en ${ }^{\circ} \mathbf{C}$} & 80 & 90 & 100 & 110 & 120 & Observations \\
\hline 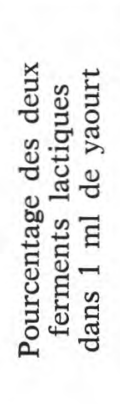 & $\begin{array}{l}\text { frais } \\
\text { congelé } \\
\text { à } \\
-40^{\circ} \mathrm{C} \\
\text { cryo- } \\
\text { desséché }\end{array}$ & $\begin{array}{l}\text { strepto. } \\
\text { lacto. } \\
\text { strepto. } \\
\text { lacto. } \\
\text { strepto. } \\
\text { lacto. }\end{array}$ & $\begin{array}{l}50 \\
50 \\
59 \\
51\end{array}$ & $\begin{array}{l}57 \\
43 \\
54 \\
46\end{array}$ & $\begin{array}{l}59 \\
41\end{array}$ & $\begin{array}{l}51 \\
49 \\
58 \\
42 \\
54 \\
46\end{array}$ & $\begin{array}{l}50 \\
50 \\
57 \\
43 \\
53 \\
47\end{array}$ & $\begin{array}{l}\text { - La méthode employée est l'exa- } \\
\text { men microscopique sur les frot- } \\
\text { tis colorés au bleu de méthy- } \\
\text { lène. } \\
\text { - Un contrôle parallèle par la mé- } \\
\text { thode de numération globale } \\
\text { avec le mélangeur de Potain et } \\
\text { la cellule de Malassez et Vignal } \\
\text { a été effectué. }\end{array}$ \\
\hline
\end{tabular}

- Le pourcentage moyen des deux ferments lactiques respectifs est de 50 p. 100 pour le streptocoque et de 50 p. 100 pour le lactobacille, dans la culture mixte fraîche.

- Le pourcentage moyen de ces deux ferments lactiques respectifs (culture mixte) congelés à $-40^{\circ} \mathrm{C}$ est de 58 p. 100 pour le streptocoque et 42 p. 100 pour le lactobacille.

- Le pourcentage moyen de ces deux ferments lactiques respectifs cryodesséchés réhydratés est de 53,6 p. 100 pour le streptocoque et 46,4 p. 100 pour le lactobacille. 


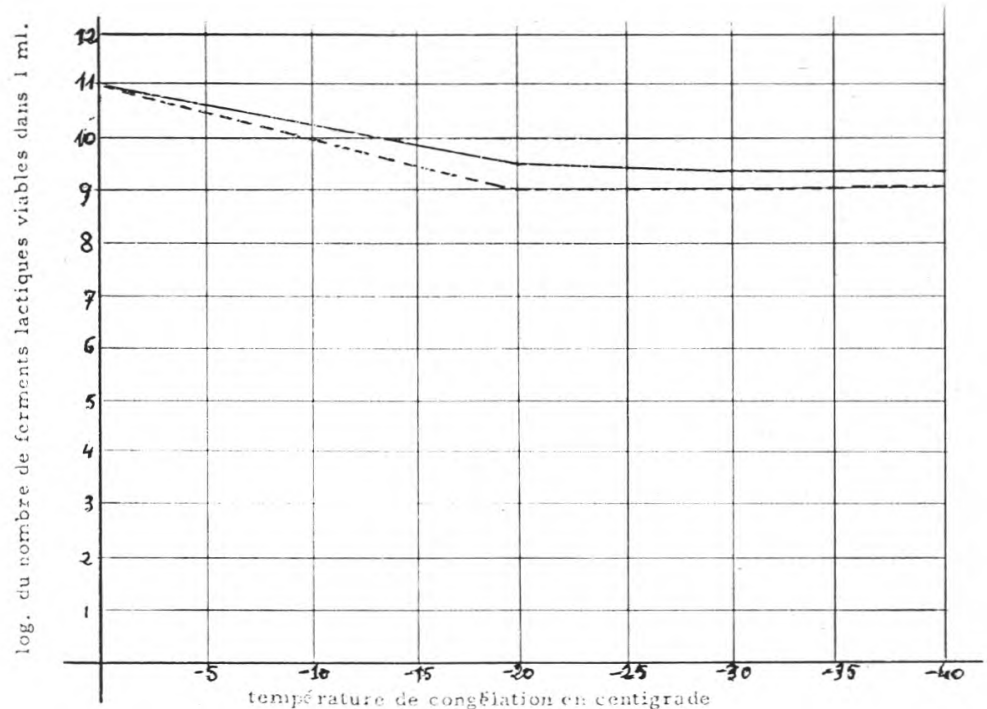

Graphique $n^{\circ} 1$

Influence des basses températures sur le taux de survie des ferments lactiques: streptocoque thermophile et lactobacille acidophile dans le yoghourt avant la phase stationnaire congelé ; - . . . . - cryodesséché

On constate que le taux de population diminue progressivement à partir de la température de congélation de $-5^{\circ} \mathrm{C}$.

La perte des ferments lactiques se stabilise à partir de $-20^{\circ} \mathrm{C}$.

Les températures très basses : $-25^{\circ} \mathrm{C},-30^{\circ} \mathrm{C},-35^{\circ} \mathrm{C}$ et $-40^{\circ} \mathrm{C}$, n'ont pas un rôle destructif sur le taux de survie de ces ferments lactiques.

Ce graphique a été établi sur la moyenne de 5 courbes correspondant pour chaque température de congélation, sauf pour le yoghourt frais dont le taux de population a été calculé sur une moyenne de 25 échantillons. 


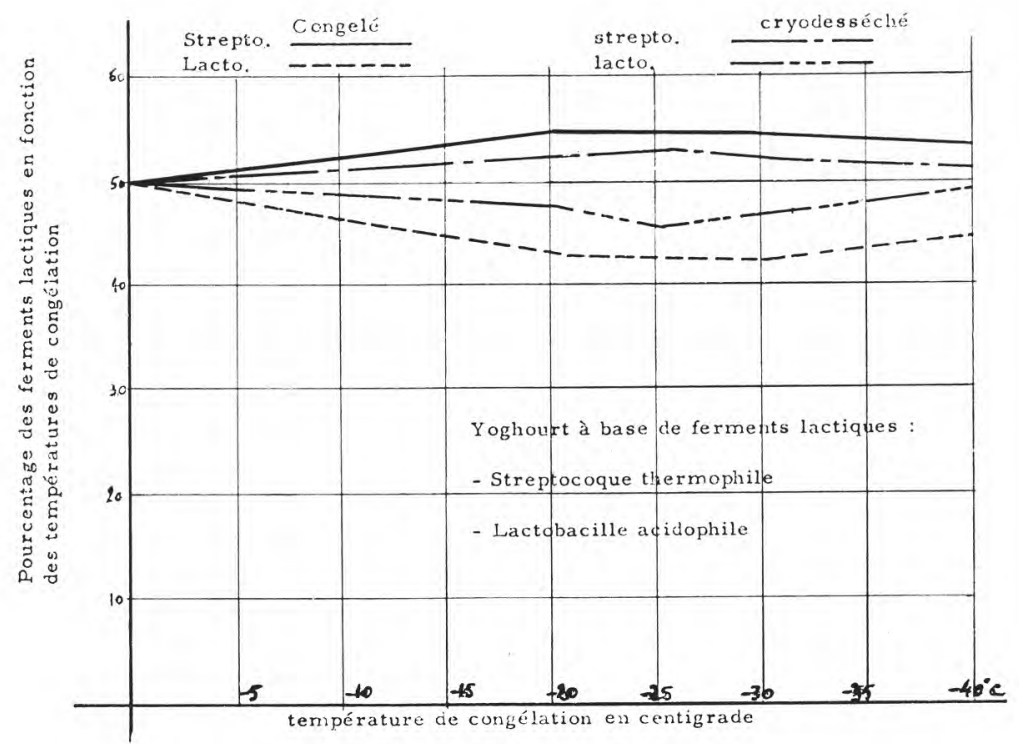

Graphique $n^{\circ} 2$

Influence des températures basses et de la cryodessiccation qui s'ensuit sur le pourcentage de deux ferments lactiques revivifiables

On constate une disproportion entre les deux ferments lactiques à partir de la température de congélation de $-5^{\circ} \mathrm{C}$.

La disproportion s'accentue au maximum à la température de congélation de $-20^{\circ} \mathrm{C}$.

La disproportion du pourcentage respectif des ferments lactiques présents se stabilise entre $-20^{\circ} \mathrm{C}$ et $-30^{\circ} \mathrm{C}$.

La disproportion du pourcentage respectif s'affaiblit à la cryodessiccation qui s'ensuit jusqu'à la température de congélation de $-30^{\circ} \mathrm{C}$.

Ce graphique a été établi sur la moyenne de 5 courbes correspondant pour chaque température de congélation, sauf pour le yoghourt frais dont le pourcentage respectif de ferments lactiques présents a été calculé sur une moyenne de 25 échantillons. 


\section{Conclusions générales}

Pour ces expériences, nous avons choisi deux ferments lactiques:

- Streptococcus thermophilus

- Lactobacillus acidophilus

pour essayer de connaître quelle était l'influence des basses températures sur le comportement physico-chimique et la viabilité de ceux-ci.

En effet, nous avons obtenu des résultats reproductibles prouvant la sensibilité des ferments lactiques étudiés, en fonction des températures de congélation.

Nous pouvons donc classer quantitativement les influences des températures basses en plusieurs catégories :

$1^{\circ}$ La perte des ferments lactiques augmente progressivement jusqu'à $-20^{\circ} \mathrm{C}$ d'une façon sensible.

$2^{\circ}$ La disproportion entre les deux ferments lactiques s'affaiblit à la température de $-30^{\circ} \mathrm{C}$, de sorte que, le pourcentage respectif des ferments lactiques présents devient presque négligeable à $-35^{\circ} \mathrm{C}$ et $-40^{\circ} \mathrm{C}$.

D'une façon générale, d'après nos expérimentations de ces dernières années qui portent sur un certain nombre de ferments lactiques tels que :

1) Streptocoque thermophile. Lactobacille bulgare.

2) Streptocoque lactique. Lactobacille acidophile.

3) Streptocoque thermophile. Lactobacille helvétique.

4) Streptocoque lactique. Lactobacille helvétique.

5) Streptocoque thermophile. Lactobacille acidophile.

Nous pouvons classer quantitativement ces ferments lactiques, suivant le taux de survie et le pourcentage respectif présents dans les milieux de cultures naturels (lait écrémé de 12 à 14 p. 100 de matière sèche) cryodesséchés en plusieurs catégories, à savoir :

$1^{\circ}$ Le taux de survie des ferments lactiques : streptocoque thermophile et lactobacille helvétique est de l'ordre de 13 à 15 p. 100.

$2^{\circ}$ Le taux de population viable des ferments lactiques : streptocoque lactique et lactobacille acidophile est de l'ordre de 12 à 13 p. 100.

$3^{\circ}$ Le taux de population viable pour les ferments lactiques : streptocoque lactique et lactobacille helvétique d'une part et streptocoque thermophile et lactobacille bulgare d'autre part est de l'ordre de 10 à 12 p. 100.

$4^{\circ}$ Le taux de population viable pour les ferments lactiques : streptocoque thermophile et lactobacille acidophile est de l'ordre de 10 p. 100.

Les températures basses à partir de $-20^{\circ} \mathrm{C}$ ont un effet nuisible sur les ferments lactiques étudiés dans des conditions de travail identique. 
La disproportion du pourcentage respectif entre les ferments lactiques présents s'affaiblit à partir de la température de congélation de $-30^{\circ} \mathrm{C}$, de sorte que, cet écart devient négligeable à la température de congélation de $-40^{\circ} \mathrm{C}$.

Les lactobacilles sont moins résistants au départ et subissent une perte énorme. Mais à partir de la température de $-25^{\circ} \mathrm{C}$ la résistance physiologique des streptocoques diminue, de sorte que l'écart de la disproportion entre les deux ferments lactiques ayant une symbiose ou une vie non symbiotique (un simple mélange de culture), s'affaiblit.

Nous pouvons donc en conclure que dans la pratique industrielle, il est préférable de congeler les cultures mixtes de ferments lactiques destinés à la cryodessiccation aux températures de congélation à partir de $-30^{\circ} \mathrm{C} /-35^{\circ} \mathrm{C}$ même à $-40^{\circ} \mathrm{C}$.

Par ailleurs, la cryodessiccation qui suit cette congélation peut améliorer, dans une certaine mesure, l'écart du pourcentage respectif des ferments lactiques présents. Celle-ci est très importante pour la réhydratation-reconstitution des cultures mixtes cryodesséchées. Son importance est de l'ordre physico-hysto-chimique, biologique et les qualités organoleptiques, à savoir : l'acidité, le $\mathrm{pH}$, la viabilité, le temps d'incubation, l'arôme, la texture, le goût, etc.

Enfin, nous pouvons suggérer aux industries alimentaires, pharmaceutiques, para-pharmaceutiques et diététiques l'emploi de cette technique de conservation des ferments lactiques. En ce qui concerne l'industrie du yoghourt (laits fermentés), en particulier, il est préférable de considérer l'emploi des ferments lactiques cryodesséchés soit pour la période de pointe, soit pour les yoghourt aromatisés, sucrés, semi-liquides, etc.

Bien entendu, la conservation des souches sous forme pure ou mixte pour longue durée en tant que levain est une des meilleures solutions pour les partisans des yoghourts.

\section{R és u m é}

D'après les résultats obtenus à la suite de nos expérimentations et en ce qui concerne l'influence des basses températures sur le taux de viabilité et le pourcentage respectif des deux ferments lactiques présents; le taux de survie et le pourcentage respectif des ferments lactiques avant et après la cryodessiccation peuvent varier de $10^{10}$ à $10^{11}$ pour le premier et de 10 à 15 p. 100 dans le milieu naturel (lait écrémé), suivant les souches de culture mixte.

\section{S u m mary}

According to result of our experimentation, concerning the effect of freezing and freeze-drying on viability and percentage of Lactic acid bacteria ; we found out that in the skin milk viability is about 
$10^{10}$ to $10^{11}$ and percentage varied between 10 to $15 \mathrm{p} .100$ following the mixte culture (symbiosis) of lactic acid bacteria.

\section{Remerciements}

Nous remercions $M$. le Professeur Keilling notre conseiller permanent, ainsi que le Professeur Ulrich pour leur concours scientifique.

Nous remercions bien vivement $M$. le Professeur Thieulin des précieux conseils qu'il a bien voulu nous donner pour la rédaction et la présentation de cet ouvrage.

\section{Bibliographie}

[1] JABARIT (A.) (1969). - Influence de la congélation et de la cryodessiccation sur le taux de survie et le pourcentage des ferments lactiques dans le yaourt (SOCALTRA, Centre de Recherche, 128, bd Victor-Hugo (92) Clichy). Le Lait, $\mathrm{n}^{\circ}$ 483-484, 160.

[2] JabARIT (A.) (1969). - Influence de la congélation et de la cryodessiccation sur le taux de survie et le pourcentage des ferments lactiques dans le bioghourt (SEPIAL, Centre de Recherche, 128, bd Victor-Hugo (92) Clichy). Le Lait, $\mathrm{n}^{\circ} 488,530$.

[3] JabaRit (A.) (1970). - Influence de la congélation et de la cryodessiccation sur le taux de survie et le pourcentage des ferments lactiques dans le yoghourt à base de ferments lactiques : Streptococcus lactis et Lactobacillus heveticus (SEPIAL, Centre de Recherche, 128, bd Victor-Hugo (92) Clichy).Le Lait, $\mathrm{n}^{\circ}$ 497, 391.

[4] JabaRIT A. (1970). - Influence de la congélation et de la cryodessiccation sur le taux de survie et le pourcentage des ferments lactiques dans le yoghourt helvétique (SEPIAL, Centre de Recherche, 128, bd Victor-Hugo (92) Clichy). Le Lait, $\mathrm{n}^{\circ} 498,544$.

[5] Orla-Jenson (S.) (1919-1943). - The lactic acid Bacterial. Copenhagen.

[6] BERgey (D. H.) (1957). - Manuel of determinative Bacteriology 7 th, edition Baltimore the, Williams and Wilkins Compagny. 